\title{
Development of an Extended Acute Care Surgery Service in Response to the Covid-19 Global Pandemic: Assessment of Clinical Patient Outcomes and Staff Psychological Well-being
}

Sachin Mathur ( $\nabla$ sachinmathur60@gmail.com )

Khoo Teck Puat Hospital https://orcid.org/0000-0003-1795-9587

Chung Fai Jeremy Ng

Singapore General Hospital

Fangju Koh

Singapore General Hospital

Mingzhe Cai

Singapore General Hospital

Gautham Palaniappan

Singapore General Hospital

Yun Le Linn

Singapore General Hospital

Huiling Linda Lim

Singapore General Hospital

Ramu Lakshman

Singapore General Hospital

Xiao Shuang Ling

Singapore General Hospital

Sock Teng Chin

Singapore General Hospital

Hiang Khoon Tan

Singapore General Hospital

Research article

Keywords: Acute care surgery, trauma, COVID-19, Singapore

Posted Date: May 15th, 2020

DOI: https://doi.org/10.21203/rs.3.rs-28816/v1 
License: (c) (i) This work is licensed under a Creative Commons Attribution 4.0 International License. Read Full License 


\section{Abstract}

\section{Background}

As the COVID-19 pandemic sweeps across the world, healthcare departments must adapt to meet the challenges of service provision and staff/patient protection. Unlike elective surgery, Acute care surgery (ACS) workloads cannot be artificially reduced providing a unique challenge for administrators to balance healthcare resources between the COVID-19 surge and regular patient admissions.

\section{Methods}

An extended ACS (eACS) model of care is described with the aim of limiting COVID-19 healthcare worker and patient cross-infection as well as providing $24 / 7$ management of emergency general surgical (GS) and trauma patients. The eACS service comprised 5 independent teams covering a rolling 1:5 24-hr call. Attempts to completely separate eACS teams and patients from the elective side were made. The service was compared to the existing ACS service in terms of clinical and efficiency outcomes. Finally, a survey of staff attitudes towards these changes, concerns regarding COVID-19 and psychological well-being was assessed.

\section{Results}

There were no staff/patient COVID-19 cross-infections. Compared to the ACS service, eACS patients had reduced overall length of stay (2-days), time spent in the Emergency Room (46 minutes) and time from surgery to discharge (2.4-hours). Mortality was decreased during this time. The eACS model of care saved financial resources and bed-days for the organisation. The changes were well received by team-members who also felt that their safety was prioritised.

\section{Conclusion}

In healthcare systems affected by COVID-19, an eACS model may assist in preserving psychological wellbeing for healthcare staff whilst providing $24 / 7$ care for emergency GS and trauma patients.

\section{Introduction}

The COVID-19 pandemic has provided humanity with enormous healthcare and economic challenges. Since Chinese New Year (CNY), the epicentre has shifted from China to Europe and the United States with over 4 million people infected and 250,000 deaths. The Singapore government escalated its 'Disease outbreak response system condition (DORCSCON) level to Orange on February 7 instigating containment strategies of isolating infected cases, aggressive contact tracing and widespread testing[1]. Despite mitigation efforts such as border closures, travel restrictions, enforced social distancing and a virtual lockdown on April 7, the number of infected cases has risen sharply to over 20,000 (3800/million) primarily via community spread. 
It was accepted that an 'all of Government, all of society' strategy was required to combat this crisis. Acute care surgery (ACS) involves managing trauma, emergency general surgery (GS) and critically ill patients. Unlike elective surgery, ACS cases cannot be cancelled during the pandemic and without appropriate strategies, ACS patients will compete for precious hospital resources such as acute ward beds, radiological tests and personal protective equipment (PPE). Furthermore, the safety and well-being of staff already working within this stressful sub-specialty comes into sharp focus[2].

Singapore General Hospital (SGH) is the leading public healthcare provider in Singapore and our ACS service has been in existence since 2016. This study describes the changes made to extend our system (eACS) to primarily safeguard against the risk of COVID-19 transmission to staff and patients, efficiently manage hospital resources and provide round the clock care. We also assessed our team-members attitudes towards the changes as well as their psychological well-being during this timeframe.

\section{Methods}

\section{ACS Model}

The ACS 'surgeon of the week' team is staffed by dedicated ACS and sub-specialty surgeons. The team admits all ACS patients during daytime hours from Monday-Friday with minimal conflict from elective surgery, endoscopy or clinics. After-hours and weekend care is provided by a roster of department surgeons with patients handed over to the ACS team in daily morning meetings. Junior staffing consists of associate consultants/fellows, senior/junior residents, non-training medical officers and house officers (interns). Typically, the team manages 20-30 admissions per day as well as 10-15 in-house referrals to GS from other medical and surgical disciplines.

\section{eACS Model}

\section{Staff management}

An eACS committee was convened after the first COVID-19 case in Singapore on Jan 23. The eACS team began admitting patients from February 3 . The primary aim of the service was to reduce exposure of surgical staff and cross-contamination of patients by COVID-19 via segregation of the department into eACS and elective. Emergency Room (ER) admissions were reviewed on the ward to limit exposure to COVID-19; only trauma activations and unstable/septic patients were seen in the ER and required donning of N95 respirator. Any ACS patient triaged as high risk for COVID-19 required full PPE: N95 respirator, hair and face shield, gown and gloves. The eACS junior staff managed ER admissions after hours and the elective team managed existing non-eACS patients with minimal interaction. Staff in clinical areas were always expected to don a surgical mask. Social distancing between team members was enforced.

The eACS service consisted of 5-teams with a rolling 1:5 24hr call system (Fig. 1). Each team was led by either an ACS surgeon or sub-specialty (Colorectal, Hepato-Biliary, Upper-Gastrointestinal) colleague who 
contributed to the service in 1-2-week blocks. The sub-specialty surgeons were drafted to ensure the eACS team had a full roster to provide $24 / 7$ care. Each team was staffed by residents, medical officers and house officers. Inter-team transfer of sub-specialty patients was allowed. Elective surgery and outpatient clinics for these teams was cancelled. Patients discharged from the eACS service were followed up by elective teams at least 2-weeks post-discharge.

\section{Protocols for admission to wards, operating room, endoscopy and radiology}

Patients were admitted from the ER to an existing ACS ward to segregate them from elective patients. COVID-19 suspected cases were admitted to the Infectious disease (ID) isolation ward. Separate acute respiratory infection 'ARI' beds were made available in multiple wards to admit surgical patients with fever, respiratory symptoms or signs of pneumonia on CXR or CT scan. In the isolation/ARI wards a combination of full registration of staff visiting and leaving the ward was required as well as donning full PPE: N95 respirator, face and hair shield, gown and gloves.

A new workflow was established for patients who required surgery (Fig. 2). An e-consent process for suspect COVID-19 cases was established with the department of Anesthesia utilising a downloaded consent form on a Toughbook@ placed in a Ziplock airtight bag. COVID-19 suspect patients required transportation by designated porters in separate elevators. An operating room (OR) with high efficiency particulate air filter (HEPA) was separated from the main OR block to reduce cross-transmission between staff/patients. All staff were to remain outside of OR during intubation and expected to don N95 respirator, face-shield and eye goggles during surgery. Low risk patients (no recent travel, contact with COVID-19 patient or fever/respiratory symptoms) underwent surgery in the main OR however anesthetic staff-maintained use of N95 respirators for intubation with standard PPE for surgeons. The protocol was tested with an in situ simulation tracking the progress of a COVID-19 positive bleeding ulcer patient with haemorrhagic shock and subsequent PEA arrest.

Stable patients that could undergo endoscopy in the outpatient setting were discharged. Urgent nonCOVID-19 eACS cases were performed in the endoscopy centre with use of PPE including N95 respirator, face-shield, gown and gloves for both upper and lower GI endoscopy based upon recommendations from in-house Gastroenterologists. This has subsequently been endorsed by the American Gastroenterological Association to protect against aerosolization of COVID-19[3]. Suspect COVID-19 patients or potentially unstable GI bleed patients underwent endoscopy in the established COVID-19 OR with full PPE.

Patients requiring urgent CT scans from the ER were transported regardless of COVID-19 status with appropriate precautions for all staff (N95 respirators). Beyond that patient workflow was determined by ward. Those in COVID-19 isolation/ARI beds were prioritised for daytime scans with protocols in place for transportation, nursing/medical escort, radiology staff PPE and necessary decontamination procedures post scan. Similar to suspect COVID-19 patients requiring OR, the patients' route from ward to CT scan was cleared and subsequently decontaminated by designated cleaning staff. Non-urgent CT scan requests were performed after 2 swab COVID-19 clearance as per ID protocol. 


\section{ACS team meetings}

All ACS meetings including journal club, trauma audit, morbidity and mortality and medical student lectures and ward-based tutorials were cancelled as per senior management directives for meetings of 10 or more people.

\section{Patients}

The study was reviewed and approved by the local Institutional Review Board under waiver of consent as anonymised data was utilised. A retrospective study was performed comparing 2-month cohorts: February/March 2019 (ACS model) and February/March 2020 (eACS model). Analysis included all ACS patients admitted through the ER and discharged under GS.

\section{Clinical and efficiency outcomes}

Prospective data collected for eACS included total number of patients seen, total in-house referrals to GS and ward round times. Data obtained from an historic time-in motion study of 10 consecutive ACS ward rounds in 2018 was used as comparison. Retrospective data obtained included demographics, surgical diagnosis and procedure (operative and endoscopy). Length of stay (LOS) was defined as duration of time between day of admission and discharge. Further time divisions identified were time from ER arrival to admission, time from CT request to CT performed for patients undergoing surgery, time from CT scan to surgery start time and time from surgery until discharge. The number of annualised bed-days saved was calculated as ((eACS mean LOS - ACS mean LOS)*number of eACS cases in 2 months)*6. Financial savings made were calculated as: number of cases*change in LOS*average ward charge and daily treatment fee. Post-operative morbidity was classified as per Clavien-Dindo[4]. Mortality data was obtained. The number of suspected COVID-19 patients was assessed as well as the total number of COVID-19 positive patients treated in SGH during the eACS time.

\section{Survey of team satisfaction with eACS and concerns regarding COVID-19}

A survey (Qualtrics, Provo. UT) was sent to all eACS staff at the end of March and appropriate consent obtained from each participant. The survey used a 5-point Likert scale to assess 3 key domains of experience: impression of eACS compared to ACS, overall concerns regarding COVID-19 and psychological wellbeing/burnout for the preceding two months. It was adapted from the Maslach Burnout Inventory which has been utilised in multiple clinical settings $[5,6]$

\section{Statistical Analysis}

Statistical analysis was performed using IBM SPSS Statistics for Windows, Version 26.0. Armonk, NY: IBM Corp. Data was analysed utilising t-test, Mann-Whitney $U$ and Chi-squared tests of comparison as appropriate. All tests were two-sided and $P<0.05$ was considered statistically significant. 


\section{Results}

\section{Clinical and efficiency outcomes}

Overall, 1436 patients were admitted during both time periods (ACS 734, eACS 702). Table 1 shows the demographics. There were no healthcare worker or patient cross-infection of COVID-19. There was a significant reduction in patients over 50 admitted to the eACS service (403 vs $469, P=0.012$ ). Similar numbers of the common-most conditions were admitted as well as operative procedures performed. There were 293 GS and sub-specialty referrals seen during the 2 months eACS. There was a decrease in total endoscopies performed ( 90 vs $121 P=0.03$ ). Overall post-operative morbidity was similar in both groups however there was a trend towards reduced mortality in the eACS era (6 vs 12 cases). The number of suspect COVID-19 cases was 96 or $14 \%$ of the admitted population. Overall SGH had treated 800 COVID-19 positive cases at the time of writing. 


\begin{tabular}{|c|c|c|c|}
\hline & $\operatorname{ACS}(n=734)$ & $\operatorname{eACS}(n=702)$ & $p$-value \\
\hline Age mean (SD) & $56(19)$ & $54(19)$ & 0.08 \\
\hline Age dichotomy $n(\%)$ & & & $0.012^{*}$ \\
\hline$\leq 50$ & $265(36)$ & $299(42)$ & \\
\hline$>50$ & $469(64)$ & $403(58)$ & \\
\hline Gender n(\%) & & & 0.55 \\
\hline Male & $371(51)$ & $366(52)$ & \\
\hline Female & $363(49)$ & $336(48)$ & \\
\hline Common conditions $n(\%)$ & & & 0.28 \\
\hline Acute appendicitis & $44(6)$ & $61(8.7)$ & \\
\hline Gall bladder disease & $87(12)$ & $81(11.6)$ & \\
\hline Intestinal obstruction & $26(3.5)$ & $33(4.7)$ & \\
\hline Skin infection/abscesses & $83(11.3)$ & $92(13.1)$ & \\
\hline GI bleed/haemorrhoids & $49(6.7)$ & $49(7)$ & \\
\hline Gastritis/peptic ulcer disease & $33(4.5)$ & $33(4.7)$ & \\
\hline Diverticulosis/diverticulitis & $38(5)$ & $32(4.5)$ & \\
\hline Trauma & $22(3)$ & $12(1.7)$ & \\
\hline Others & $352(48)$ & $309(44)$ & \\
\hline Operative procedures performed $\mathrm{n}(\%)$ & $171(23)$ & $179(25)$ & NS \\
\hline Clavien-Dindo 3+ & 4 & 6 & NS \\
\hline
\end{tabular}

SD Standard deviation

$n=$ number of observations

* $P<0.05$

NS; not significant, HCW, Healthcare worker

Others refers to a combination of non-specific abdominal pain, ureteric colic, urinary tract infection or other medical, oncological, urological, gynecological and orthopedic disorders

Trauma activations refers to total trauma cases attended to in the ER, however some are subsequently admitted to Orthopedic surgery and Neurosurgery 


\begin{tabular}{|c|c|c|c|}
\hline & $\operatorname{ACS}(n=734)$ & eACS $(n=702)$ & $p$-value \\
\hline Endoscopies performed n(\%) & $121(16)$ & $90(13)$ & $0.03^{\star}$ \\
\hline Inpatient referrals seen (GS and sub-specialty) & N.A. & 293 & \\
\hline Total trauma activations & 39 & 30 & 0.36 \\
\hline Mortality & $12(1.6)$ & $6(0.9)$ & 0.18 \\
\hline COVID-19 Suspected:Confirmed cases & 95:0 & & \\
\hline COVID-19 positive cases treated in SGH & 800 & & \\
\hline Patient/HCW infection with COVID-19 & & 0 & \\
\hline \multicolumn{4}{|l|}{ SD Standard deviation } \\
\hline \multicolumn{4}{|l|}{$n=$ number of observations } \\
\hline \multicolumn{4}{|l|}{$* P<0.05$} \\
\hline \multicolumn{4}{|l|}{ NS; not significant, HCW, Healthcare worker } \\
\hline \multicolumn{4}{|c|}{$\begin{array}{l}\text { Others refers to a combination of non-specific abdominal pain, ureteric colic, urinary tract infection or } \\
\text { other medical, oncological, urological, gynecological and orthopedic disorders }\end{array}$} \\
\hline $\begin{array}{l}\text { Trauma activations refers to total trauma cases } \\
\text { subsequently admitted to Orthopedic surgery an }\end{array}$ & $\begin{array}{l}\text { led to in the ER, } \\
\text {-osurgery }\end{array}$ & wever some are & \\
\hline
\end{tabular}

The LOS decreased by 2 days from ACS to eACS period [mean(SD) 5.2(14.5) vs 3.2(3.0), $P<0.0001$ ]. The ER arrival to admit time decreased by 46 minutes [median (range) $2.0 \mathrm{~h}(0.1-20.4)$ vs $1.4(0.2-12.2), P<$ 0.001]. For patients undergoing surgery, the time from CT scan ordered to CT performed reduced but was not significant [median (range) $5.0 \mathrm{~h}(0.77-36.7)$ vs $4.5 \mathrm{~h}(0.6-38.2)$ ]. There was a non-significant reduction in time from CT scan to surgery [median (range) $13.8 \mathrm{~h}(0.12-255)$ vs $7.8 \mathrm{~h}(0.35-222)$ ]. However, the time from surgery to discharge decreased significantly by 2.4 hours [median (range) $38.8 \mathrm{~h}(7.9-640)$ vs $36.4 \mathrm{~h}(5.7-409), P=0.001]$. The annualised eACS bed-days saved was 8424 . The LOS reduction was calculated to save the institution $\$ 222,632$ Singapore dollars over 2-months.

Figure 3 shows the ward distribution for admitted patients. There was a $29 \%$ increase in patients admitted to the surgical wards during eACS (overall $75 \%$ vs $58 \%, P<0.001$ ). Of the eACS patients $14 \%$ were admitted to ARI/Isolation wards. Compared to the historical control of 86 minutes, eACS average ward rounds across 5 -teams were $35 \%$ shorter (56 minutes).

\section{Survey of team satisfaction with eACS and concerns regarding COVID-19}

The survey was answered by $90 / 92$ members of the eACS team ( $98 \%$ response rate). 
When comparing eACS to ACS, the team members enjoyed working within the new system (Fig. 4a). Specifically, the majority felt they were able to maintain rostered junior hours and focus on patient care. They also felt the service provided timely access to scans and surgery for eACS patients.

With regard to COVID-19, there was a clear pattern of concern amongst staff that they were at risk of contracting the disease and passing it onto their family members (Fig. 4b). Conversely, there was near universally acceptance that the system provides appropriate protections and allows for segregation of healthcare workers.

The specific questions and answers for burnout/psychological wellbeing are shown in Fig. 4c. The vast majority of respondents felt appreciated, that they were positively influencing patients' lives and they were accomplishing worthwhile things at work. Questions pertaining to frustration with work or feeling emotionally drained or fatigued were met with almost universal disagreement.

\section{Discussion}

This study describes the rapid development of an eACS service in response to the COVID-19 outbreak. There were no team-member or patient cross-infections from COVID-19 during the study period. The eACS model resulted in improved efficiency of care, clinical outcomes and reduced costs compared to the previous ACS service. Team morale and satisfaction with the service remained high despite concerns about contracting COVID-19 or passing it on to family members.

\section{The need for an eACS model (preparedness)}

After the SARS outbreak of 2003 where 5 healthcare workers lost their lives in Singapore, preparedness models were created for future outbreaks[7]. Senior management dictated a zero tolerance for healthcare worker infection. By separating eACS and elective streams within the department and adapting the service to 5 teams, we prevented its collapse secondary to individual infection or quarantine. A similar approach was utilised by Ngoi et al in managing Oncology patients by adapting their department into 2 separate teams[8]. Further limitations such as restricted access to ER and inter-team meetings/social gatherings facilitated this result. Furthermore, by reducing costs we could potentially divert vital resources towards maintaining PPE/ventilators for those on the front-line.

Early co-ordination with the ID, ER, OR, porters and cleaning departments were able to protocolise the movement of suspect COVID-19 patients to limit staff exposure. This has been reinforced in recent global surgical guidelines for COVID-19[9]. Protocols for PPE utilisation were developed to minimise wastage of resources. The use of in situ simulation enabled aspects of team dynamics and protocols to be tested in a safe environment and has been used extensively in surgical settings[10,11]. We were able to modify workflows surrounding blood transfusion requests, patient transportation and time required to setup COVID-19 OR. Furthermore the establishment of a designated leader (OR Anesthetist) to streamline communication between staff members and co-ordinate the logistics of transfer was encouraged with the aim of reducing the time from OR activation until patient arrival to 20 minutes[12]. Ross et al in their 
multi-tiered response to COVID-19 placed the ACS team front and centre with ACS staff transitioned to ICU whereas emergency GS and trauma cases were handled by suitably trained faculty[13]. In times of overwhelming COVID-19 admissions, it would be an appropriate consideration in Singapore.

\section{eACS clinical and efficiency outcomes}

ACS models of care have consistently shown improved outcomes including LOS, costs, reduced complications and mortality[14-18]. Our eACS model reduced LOS further with significant cost savings to the institution in just 2-months. Furthermore, the bed-savings made would assist with preparing wards for the expected COVID-19 surge. The decreased LOS was achieved through multiple pathways:

1.

Reduced admission times for surgical patients in the ER will allow for more beds for COVID-19 suspect patients.

2.

The dedicated ACS ward (not present in 2019) provided a natural home for admitted patients, with nurses and ancillary medical staff attuned to their needs. The reduced ward round times highlighted the efficiencies that can be achieved.

3.

Sequestering suspect COVID-19 patients to ARI/isolation wards expedited care for these patients who were prioritised for swab clearance, $\mathrm{CT}$ scans and $\mathrm{OR}$ if required.

4.

Rapidly established workflow with radiology and OR/Anesthesia was achieved highlighted by the reductions in time from CT scan to surgery and from surgery to discharge for eACS patients.

5.

Our laboratory partners developed a novel COVID-19 test which enabled accelerated clearance of suspect patients within hours. It is a reverse transcription real time polymerase chain reaction (RT-PCR) targeting the E-gene of COVID-19. The assay was developed based upon published protocols[19].

The overall workload between both time-periods was similar but also consistent for the downward fluctuation that occurs in February because of CNY. Fewer older admissions were noted and likely related to government directives to stay at home. Fewer endoscopy cases related to stable patients being offered outpatient evaluation. Reduction in trauma cases were noted which may be related to a combination of government DORSCON orange status, stay at home mandates and lockdown. One aspect of workflow which did increase was inter-team transfers and reviews by the sub-specialist surgeons. This was expected since these reviews were not seen by elective teams anymore. The junior manpower of the eACS service was reduced from February to March to reflect the reduction in admissions noted.

\section{Staff satisfaction and morale}

The eACS service was well received by the vast majority of the team. The structure of the service leads to 'off-days' during which personal time to de-stress appears to be helping. Whilst not a complete picture of the mental state of the team, the results are encouraging given that up to $61 \%$ of US surgical residents 
exhibited burnout on at least one of three sub-scales: emotional exhaustion, depersonalisation and personal accomplishment[20].

It is clear that concerns for personal safety and the health of loved ones is prevalent amongst the teammembers. However, the survey also shows that they felt the system prioritised their safety, which was one of our key goals. This has likely contributed to a positive psychological well-being as assessed in the survey. An appropriate level of PPE is vital and out of the control of most clinicians hence reliance upon the institution and government will always be there.

Support systems are imperative for healthcare workers both at home and at the institution level. SGH has multiple links for staff to access psychological support mechanisms in-house. The World Health Organisation has advocated for de-stigmatising healthcare workers from abuse in the community, encouraging workers to avoid unhelpful coping strategies and remembering that it's a marathon not a sprint[21].

\section{Limitations}

A limitation of this study is the generalisability of the eACS model. When consistent attempts to 'flatten the curve' are made, this eACS strategy may assist to limit staff and patient exposure to COVID-19. However, for our heroic colleagues working in healthcare systems decimated by COVID-19, they will have their own unique strategies to deal with ACS cases and we look forward to learning from them. This pandemic is far from over and adaptability of systems will be the key to success. Our strategy will need to be re-examined in future studies beyond COVID-19.

\section{Conclusions}

This is the first study to report results after the implementation of an extended ACS model of care in response to the COVID-19 global pandemic. We have shown improved efficiency of care and clinical outcomes for these patients. Furthermore this study uniquely addresses the psychological well-being of our team-members in response to the pandemic and highlights the need for robust systems to protect workers/patients from cross-infection.

\section{Abbreviations}

COVID-19

Coronavirus disease 19

ACS, eACS

Acute care surgery, extended Acute Care Surgery

$\mathrm{SGH}$

Singapore General hospital

GS

General surgery 
CNY

Chinese New Year

DORSCON

Disease outbreak response system condition

PPE

Personal protective equipment

ER

Emergency Room

OR

Operating Room

ID

Infectious disease

ARI

Acute respiratory infection

GI

Gastro-intestinal

CT

Computerised tomography

LOS

Length of stay

RT-PCR

Reverse transcriptase polymerase chain reaction

\section{Declarations}

\section{Ethics approval and consent to participate}

Clearance was obtained from the Institutional Review Board with exemption provided for consent as anonymised data was utilised

\section{Consent for publication}

Obtained from each author, anonymised data was used hence exemption was obtained for study patients

\section{Availability of data and materials}

If required we can provide data as needed

\section{Competing interests}


None

\section{Funding}

No funding source obtained for this work

\section{Authors Contributions}

Sachin Mathur - Study design, analysis, interpretation, drafting, revising, final approval

Jeremy $\mathrm{Ng}$ - Analysis, interpretation, revising, final approval

Fangju Koh - Study design, analysis, revising

MingZhe Cai - Study design (survey), analysis

Gautham Palaniappan - analysis, interpretation

Yun Le Linn - analysis, interpretation

Huiling Linda Lim - analysis, interpretation

Ramu Lakshman - analysis, interpretation

Xiao Shuang Ling - study design (survey), revising

Sock Teng Chin - analysis, interpretation, revising

HiangKhoon Tan - study design, drafting, final approval

\section{Acknowledgments}

The study acknowledges Mr Hong Wei Tan from the Department of Strategy Management and Analytics, SGH for assistance with data collection, analysis and costs calculation.

\section{References}

1. $\mathrm{MOH}$. MOH pandemic readiness and response plan for influenza and other acute respiratory diseases. Available from https://www.moh.gov.sg/docs/librariesprovider5/diseases-updates/interimpandemic-plan-public-ver-april-2014.pdf. 2014.

2. Brindle M, Gawande A. Managing COVID-19 in Surgical Systems [published online ahead of print March 23 2020]. Ann Surg. 2020. 
3. Sultan S, Lim JK, Altayar O, Davitkov P, Feuerstein JD, Siddique SM, et al. AGA Institute Rapid Recommendations for Gastrointestinal Procedures During the COVID-19 Pandemic. Gastroenterology. 2020.

4. Dindo D, Demartines N, Clavien PA. Classification of surgical complications: a new proposal with evaluation in a cohort of 6336 patients and results of a survey. Ann Surg. 2004;240(2):205-13.

5. Kemper KJ, Wilson PM, Schwartz A, Mahan JD, Batra M, Staples BB, et al. Burnout in Pediatric Residents: Comparing Brief Screening Questions to the Maslach Burnout Inventory. Academic pediatrics. 2019;19(3):251-5.

6. Rotstein S, Hudaib AR, Facey A, Kulkarni J. Psychiatrist burnout: a meta-analysis of Maslach Burnout Inventory means. Australasian Psychiatry. 2019;27(3):249-54.

7. Goh KT, Cutter J, Heng BH, Ma S, Koh BK, Kwok C, et al. Epidemiology and control of SARS in Singapore. Annals of the Academy of Medicine, Singapore. 2006;35(5):301-16.

8. Ngoi N, Lim J, Ow S, Jen WY, Lee M, Teo W, et al. A segregated-team model to maintain cancer care during the COVID-19 outbreak at an academic center in Singapore. Annals of Oncology. 2020;20:36410-3.

9. Collaborative C. Global guidance for surgical care during the COVID-19 pandemic. BJS (British Journal of Surgery).[published ahead of print] doi:10.1002/bjs.11646.

10. Owei L, Neylan CJ, Rao R, Caskey RC, Morris JB, Sensenig R, et al. In Situ Operating Room-Based Simulation: A Review. Journal of Surgical Education. 2017;74(4):579-88.

11. Minor S, Green R, Jessula S. Crash testing the dummy: a review of in situ trauma simulation at a Canadian tertiary centre. Can J Surg. 2019;62(4):243-8.

12. Wong J, Goh QY, Tan Z, Lie SA, Tay YC, Ng SY, et al. Preparing for a COVID-19 pandemic: a review of operating room outbreak response measures in a large tertiary hospital in Singapore. Can J Anaesth. 2020:1-14.

13. Ross SW, Lauer CW, Miles WS, M. GJ, Christmas AB, May AK, et al. Maximizing the Calm Before the Storm: Tiered Surgical Response Plan for Novel Coronavirus (COVID-19). J Am Coll Surg.20:30263-5.

14. Murphy PB, DeGirolamo K, Van Zyl TJ, Allen L, Haut E, Leeper WR, et al. Impact of the Acute Care Surgery Model on Disease- and Patient-Specific Outcomes in Appendicitis and Biliary Disease: A Meta-Analysis. J Am Coll Surg. 2017;225(6):763-77.e13.

15. Ball CG, MacLean AR, Dixon E, Quan ML, Nicholson L, Kirkpatrick AW, et al. Acute care surgery: the impact of an acute care surgery service on assessment, flow, and disposition in the emergency department. Am J Surg. 2012;203(5):578-83.

16. Hoyt DB, Kim HD, Barrios C. Acute care surgery: a new training and practice model in the United States. World J Surg. 2008;32(8):1630-5.

17. Mathur S, Lim WW, Goo TT. Emergency general surgery and trauma: Outcomes from the first consultant-led service in Singapore. Injury. 2018;49(1):130-4. 
18. Mathur S, Goo TT, Tan TJ, Tan KY, Mak KS. Changing models of care for emergency surgical and trauma patients in Singapore. Singapore Medical Journal. 2016;57(6):282-6.

19. Corman VM, Landt O, Kaiser M, Molenkamp R, Meijer A, Chu DKW, et al. Detection of 2019 novel coronavirus (2019-nCoV) by real-time RT-PCR. Euro Surveill. 2020;25(3).

20. Elmore LC, Jeffe DB, Jin L, Awad MM, Turnbull IR. National Survey of Burnout among US General Surgery Residents. J Am Coll Surg. 2016;223(3):440-51.

21. WHO. Mental health and psychosocial considerations during the COVID-19 outbreak, 18 March 2020. World Health Organization; 2020.

\section{Figures}

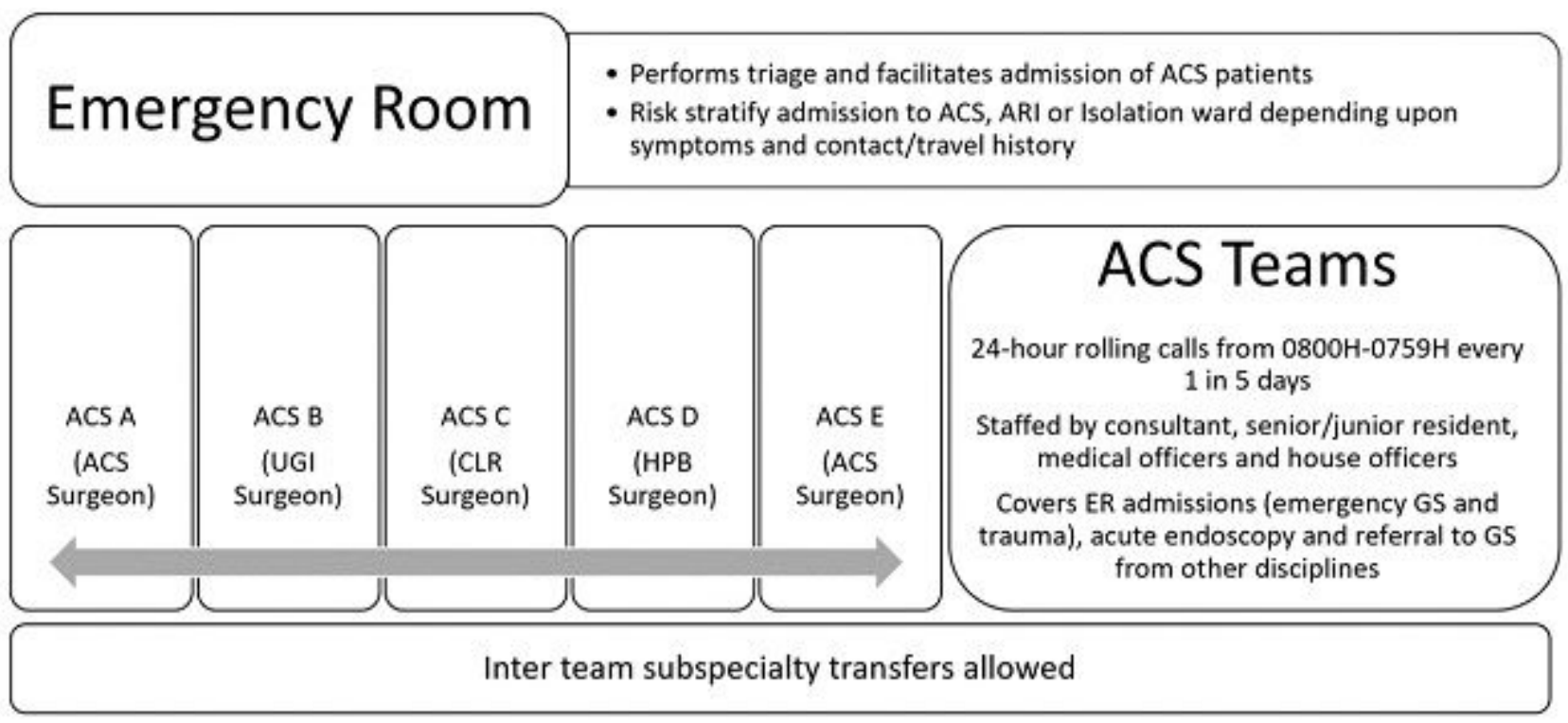

Abbrev: ACS; Acute care surgery, ARI; Acute respiratory infection, UGI; Upper gastro-intestinal surgeon, CLR; Colorectal surgeon, HPB; Hepato-biliary surgeon, GS; General surgery, ER; Emergency Room

\section{Figure 1}

Enhanced ACS service from February 3, 2020 


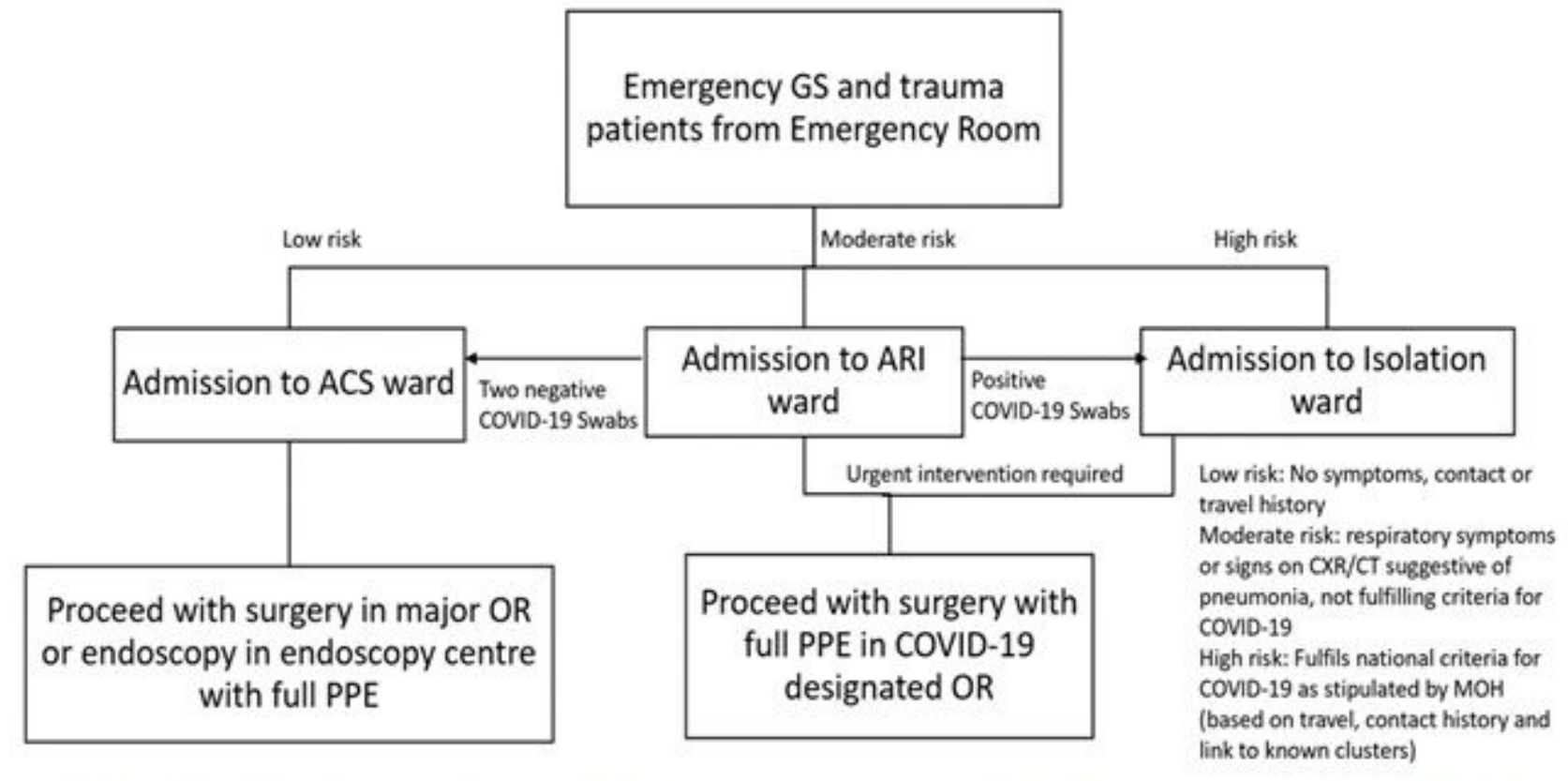

Abbreviations: GS; General Surgery, ACS; Acute care surgery, ARI; Acute respiratory infection, OR;

operating room, PPE; personal protective equipment, CXR; Chest X-ray, CT; Computed Tomography, $\mathrm{MOH}$; Ministry of Health

\section{Figure 2}

Workflow for eACS patients admitted from ER requiring surgery/endoscopy

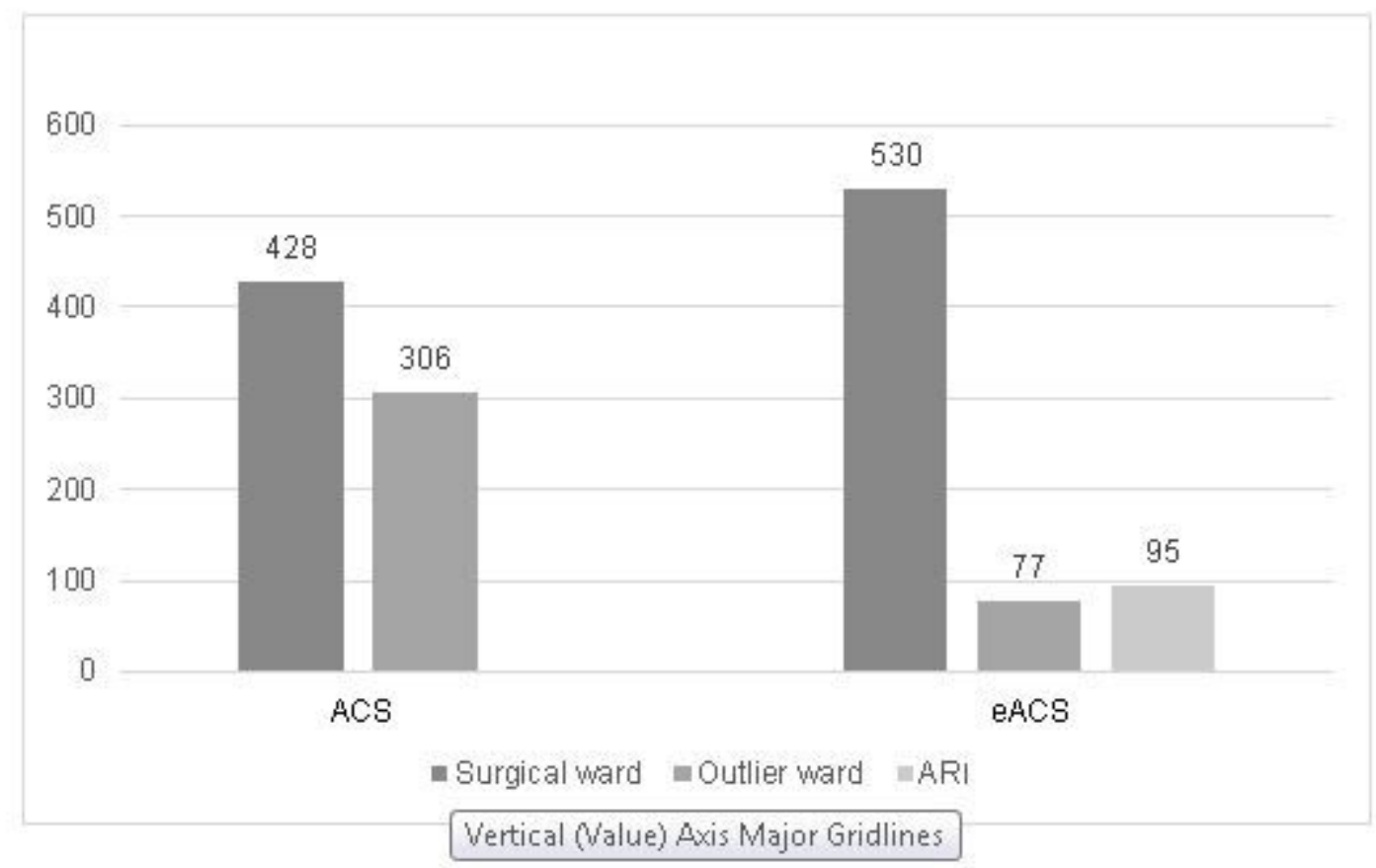

Abbreviation: ARI; acute respiratory ward 
Figure 3

Ward distribution for ACS and eACS patients
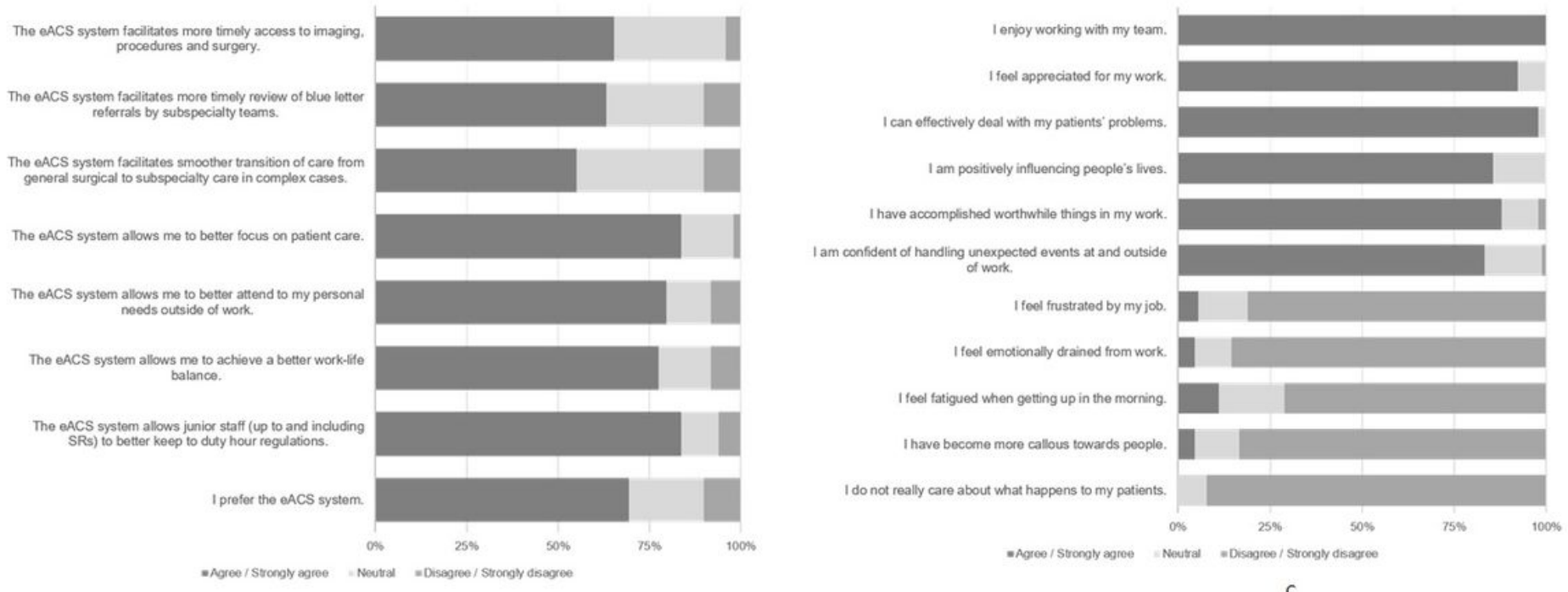

A

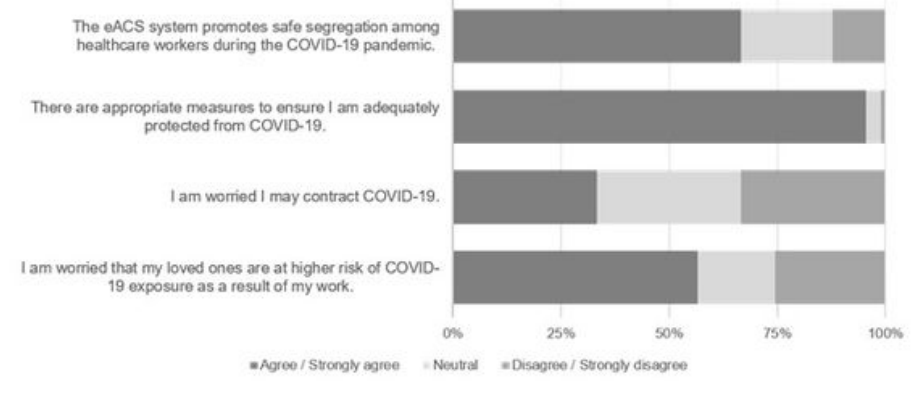

B

\section{Figure 4}

a Survey responses to questions differentiating ACS and eACS systems b Survey responses to questions regarding COVID-19 concerns and safety at work c Survey responses to questions specifically related to burnout and psychological well-being 